\title{
Escolaridade nas zonas rurais da região sul
}

Schooling in rural areas of the southern region

La escolarización en las zonas rurales de la región sur.

Scolarité dans les zones rurales de la région sud

\section{Amarildo de Paula Junior}

\section{OpenEdition}

\section{Journals}

\section{Edição electrónica}

URL: http://journals.openedition.org/espacoeconomia/9900

DOI: 10.4000/espacoeconomia.9900

ISSN: 2317-7837

\section{Editora}

Núcleo de Pesquisa Espaço \& Economia

\section{Refêrencia eletrónica}

Amarildo de Paula Junior, «Escolaridade nas zonas rurais da região sul », Espaço e Economia [Online], 16 | 2019, posto online no dia 03 janeiro 2020, consultado o 10 janeiro 2020. URL : http:// journals.openedition.org/espacoeconomia/9900 ; DOI : 10.4000/espacoeconomia.9900

Este documento foi criado de forma automática no dia 10 janeiro 2020.

(c) NUPEE 


\title{
Escolaridade nas zonas rurais da região sul
}

\author{
Schooling in rural areas of the southern region \\ La escolarización en las zonas rurales de la región sur. \\ Scolarité dans les zones rurales de la région sud
}

Amarildo de Paula Junior

\section{Introdução}

1 O PIB do agronegócio brasileiro de acordo com o Centro de Estudos Avançados em Economia Aplicada - CEPEA em 2017 foi de 1.418,778 milhões a preços correntes e representou $21,6 \%$ do PIB total do país, denotando a magnitude da importância do setor para a economia nacional. Esse ano também foi marcado pelo clima favorável para o crescimento recorde das safras, atingindo novos níveis de produção e produtividade.

2 Contudo, no sentido inverso desse crescimento o número de pessoas ocupadas no setor do agronegócio apresentou redução em 2017. Sendo que em 2016 o número de ocupados no setor foi de 18,53 milhões de pessoas e já em 2017 foi de 18,24 milhões, queda 1,6\%. Fechando o ano de 2017 com 20,1\% de participação no total de ocupados no país, ainda assim de forma significativa o setor foi responsável por boa parte dos postos de trabalho disponibilizados na economia.

3 Observada essa redução no número de ocupados no mercado de trabalho do agronegócio, a questão é o que está ocorrendo no segmento do agronegócio brasileiro e que pode ser respondida pelo relatório de Mercado de Trabalho compilado pelo CEPEA em 2018, que aborda sobre a redução nos postos de trabalho para aquelas pessoas que possuem pouca ou nenhuma instrução, tornando-as vulneráveis e que outrora ocupavam as vagas no setor primário.

4 Ocorre que com a atualização constante de novas tecnologias que vão sendo implementadas no agronegócio para ganho de tempo e eficiência, a mão de obra necessária se reduz e é requisitada cada vez mais com um alto nível de sofisticação. $\mathrm{E}$ 
ainda aqueles produtores que possuem baixa dotação de capital humano, mas que são produtores independentes, dado o contexto de inovação contínua acabam por serem suprimidos pelo meio concorrencial (CEPEA, 2018).

5 A agricultura por ser na em grande parte das vezes de característica familiar, está repleta de trabalhadores com baixa escolaridade. No estudo de Hoffmann e Gomes Ney (2004) é observado que a agricultura tem nível de escolaridade menor do que outros setores da economia brasileira, como o setor de serviços e de indústria. 0 fato do nível de escolaridade na atividade primária ser inferior ao nível de escolaridade alocado nas atividades secundárias e terciárias deve estar relacionado ao fato de maior demanda por mão-de-obra qualificada em atividades como construção civil, serviços e indústria.

6 Porém, com o avanço de tecnologias se fez necessário a implementação de novos processos de produção com a utilização de máquinas e equipamentos mais aprimorados. Essas novas formas de se produzir causou um ligeiro deslocamento de mão-de-obra qualificada para a atividade primária, de maneira que a proporção: agricultores sem escolaridade/total de agricultores veio a reduzir ligeiramente.

7 Compreendido a importância do setor do agronegócio para a economia brasileira, e da formação de capital humano para a sequência de avanços tecnológicos que vem sendo feitos no setor, e para a manutenção dos postos de trabalho e a possível criação de novos, é relevante que o tema seja abordado. Esforço esse que pode ser pensado como uma forma de mitigar o que Stulp (2006) descreve como sendo uma migração ruralurbana, e que, "com o avanço tecnológico nos outros setores que não o agrícola, as dificuldades para a mão de obra rural, não treinada, encontrar emprego nos mesmos, se torna cada vez maiores", assim esse estudo abrange o efeito que a escolaridade das pessoas que residem e trabalham na área rural provocam sobre a produção agrícola, e ao mesmo tempo parte da hipótese de que esse efeito seja positivo, sendo a região Sul e seus principais produtos agrícolas os selecionados para análise entre o período de 2001 a 2015.

8 O estudo está estruturado em cinco seções, além dessa introdução, a seção 2 trata da revisão da literatura sobre a influência do capital humano empregado em zonas rurais sobre sua produção agrícola. A seção 3 apresenta a abordagem metodológica e os modelos de estimação. A seção 4 apresenta os resultados alcançados e, por fim, a última seção corresponde às principais conclusões do artigo.

\section{Revisão de literatura}

9 As raízes da discussão literária para a tentativa de medir e compreender a formação do capital humano se origina com os economistas clássicos, como Adam Smith (1776), com a sua obra "A Riqueza das Nações", e Alfred Marshall (1920), com "Os princípios econômicos do mais valioso investimento em capitais, os seres humanos", tendo ricas contribuições oriundas da Escola de Chicago, com Gary Becker, Jacob Mincer e Theodore Schultz (1902-1998), este último foi o responsável por elaborar a expressão teoria do capital humano e expô-la na década de 60 (CABRAL et ali, 2016).

10 Teve ainda importantes contribuições com o trabalho de Lucas (1988) que segundo Ferreira e Veloso (2005), o argumento de Lucas sobre a escolaridade além de ter um impacto direto no produto também traz uma externalidade positiva, visto que a produtividade dos trabalhadores também tende a aumentar como se fosse um ciclo benéfico. 
11 Observando os efeitos do capital humano na produtividade, a ênfase será atribuída ao meio rural, objeto deste estudo. Mendes e Pereira (2009) afirmam que a educação quando inserida no meio rural seria uma ferramenta para introduzir conhecimento e habilidades que transformaria a realidade da economia de subsistência que muitas vezes está em zonas rurais em uma economia mais desenvolvida e moderna.

Durante a década de 1990 grande parte da mão-de-obra da agricultura brasileira era composta por trabalhadores temporários com baixa escolaridade. Entretanto, o novo ciclo tecnológico que segundo Staduto, Shikida e Bacha (2004) fez emergir máquinas e equipamentos poupadores de mão-de-obra, como máquinas de corte e limpeza de canade-açúcar, além de colheitadeiras de algodão e café. Isto tornou indispensável a elevação de escolaridade de pessoas que estão alocadas no meio rural para que se mantenham ocupadas no mercado de trabalho.

13 De acordo com Lewis apud Stulp (2006), com essa introdução da educação no meio rural, haverá na agricultura uma oferta de mão de obra ilimitada com produtividade marginal do trabalho tendendo a zero, isto é, acrescentando uma unidade a mais de mão de obra está tenderia a render uma proporção menor de trabalho que a unidade anterior. E essa mão de obra migraria para os centros urbanos dada a escassez de oferta de trabalho no campo. Porém mais tarde Lewis revisa sua abordagem, pontuando que a agricultura não deve ser passiva quanto as mudanças iminentes, e sim deve investir em programas de extensão rural, incentivar o cooperativismo, gerar desenvolvimento agroindustrial e prover capacitação tecnológica para o produtor rural, com o objetivo de reduzir a migração rural-urbana.

14 Para Schultz (1973) existe uma série de fatos que demonstram que a educação tem uma relação com a produtividade, exemplificado pelo autor com os fenômenos da recuperação do pós-guerra na Europa, ou na dificuldade de países em desenvolvimento em absorver adequadamente capitais importados, fato este corroborado por Ferreira e Veloso (2005) que enfatizam que "um nível mais elevado de escolaridade da força de trabalho facilita a absorção de novas tecnologias", facilitando assim o desenvolvimento dos indivíduos e da economia.

Assim a teoria do capital humano difundida por Schultz, enfatizava que a melhoria do bem-estar das pessoas desprovidas de acesso a terras, maquinas e, ou equipamentos, não derivava destes últimos, mas sim do conhecimento. Portanto, considera-se que todas as habilidades são inatas ou adquiridas e devem ser aperfeiçoadas, se utilizando de métodos que levem a esse aprimoramento intelectual (CABRAL et al, 2016).

Para Mendes e Pereira (2009), o ponto a ser discutido acerca de capital humano está na qualidade da mão de obra e não na quantidade de capital ofertado, sendo físico ou humano (uso intensivo de mão de obra), ou seja, de nada adianta ter maquinas e equipamentos avançados tecnologicamente, se não há mão de obra especializado apta a operá-los.

17 Sendo assim, para Daniel Cotlear (1989) apud Mendes e Pereira (2009) existe três efeitos da educação sobre a produtividade agrícola, (i) o efeito sobre o trabalhador, este aprimora a qualidade do trabalho e faz com que o produtor possa auferir maiores quantidades usando a mesma quantidade de insumo, (ii) o efeito de alocação, este permite que o produtor se torne mais apto a processar informações outrora não distinguíveis, por ele e (iii), o efeito de seleção de insumos, que melhora no curto prazo a escolha dos insumos e no longo prazo a escala de operação. 
18 Ainda para Mendes e Pereira (2009), estes abordam sobre a segmentação do mercado de trabalho, e enfatizam que a influência do nível educacional é de suma importância para a mobilidade entre os segmentos do mercado. Portanto, o esforço em diminuir as fronteiras do conhecimento é percebido como uma busca extremamente racional.

19 Esse esforço, ou busca racional empreendido pelos indivíduos e posteriormente pelo coletivo, em investir em capital humano, para usufruir de um retorno positivo no futuro, se traduz num fator elementar para ampliar a produtividade e mitigar o atraso econômico de uma nação (CABRAL et al, 2016).

20 No arcabouço da agricultura nacional, já começa a apresentar exigência de escolaridade para trabalhadores de zonas rurais, como explica Silva (2001). Em algumas regiões para a contratação de mão-de-obra para agricultura, se exige no mínimo quatro anos de estudo para que esteja apto a trabalhar no corte de cana e o dobro caso esteja interessado em ser redirecionado para o trabalho em usinas. Essa exigência que pode ser motivo de controvérsia, no longo prazo pode trazer consequências positivas sobre a produtividade agrícola da região, resultando em geração de empregos e renda.

21 Visando o setor do agronegócio como de extrema importância para a economia brasileira, no seu processo de retomada do crescimento, é pertinente que sejam desenvolvidas políticas direcionadas a esse objetivo. Dessa forma, Johnston e Mellor (1961) afirmaram que a renda oriunda do setor agrícola tenderia a elevar-se para consolidar num setor de mercado agroindustrial e por capitalização gerar uma maior oferta de alimentos, visto que no momento em que a renda dos outros setores aumenta, a renda do agronegócio deverá aumentar também.

\section{Escolaridade nas zonas rurais do Paraná, Santa Catarina e Rio Grande do Sul}

22 Além da produtividade agrícola e aumento da renda, o capital humano empregado em zonas rurais também aprimora a utilização de terras e equipamentos. Goméz (2007) aborda sobre a melhor utilização de recursos limitados como o território destinado à plantação e políticas públicas voltadas para esse assunto, o que corrobora Kageyama (2003). Em seu estudo que analisa a produtividade agrícola de famílias que participam do PRONAF, os resultados obtidos apontam que os participantes que possuem uma maior escolaridade têm uma maior produtividade agrícola, devido ao uso de um nível maior de tecnologia química e mecânica comparada à famílias com menor escolaridade.

23 A convergência de desenvolvimento territorial causada pelo capital humano não somente ocorre à nível nacional, como também pode ocorrer em zonas rurais isoladas. Barro (1991) explica que para um determinado nível escolaridade de uma região pobre, ao longo do tempo essa escolaridade pode reduzir a diferença no desenvolvimento comparada à uma região mais rica. No entanto, se as zonas rurais se desenvolverem de forma tão acelerada que atinja o patamar de zonas urbanas, o território disponibilizado à produção agrícola poderá ser destinado a outro fim, como a instalação de uma indústria de manufatura ou construção de moradias.

24 A figura 1 apresenta o número de pessoas dos estados da região Sul que concluíram ao menos o ensino médio e, que residem e trabalham em zonas rurais. Os estados do Rio Grande do Sul e do Paraná alternaram entre si no posto de estado com o maior número dessas pessoas. Após 2010 houve uma redução relevante no número dessas pessoas no Rio Grande do Sul, o que pode ser explicado pela migração para o meio urbano. Já o 
estado de Santa Catarina é o que apresenta o menor número dessas pessoas, embora tenha quase triplicado esse montante entre 2001 e 2015.

Figura 1 - Número de residentes de zonas rurais dos estados da região Sul que trabalham com agricultura e possuem ao menos o ensino médio completo.

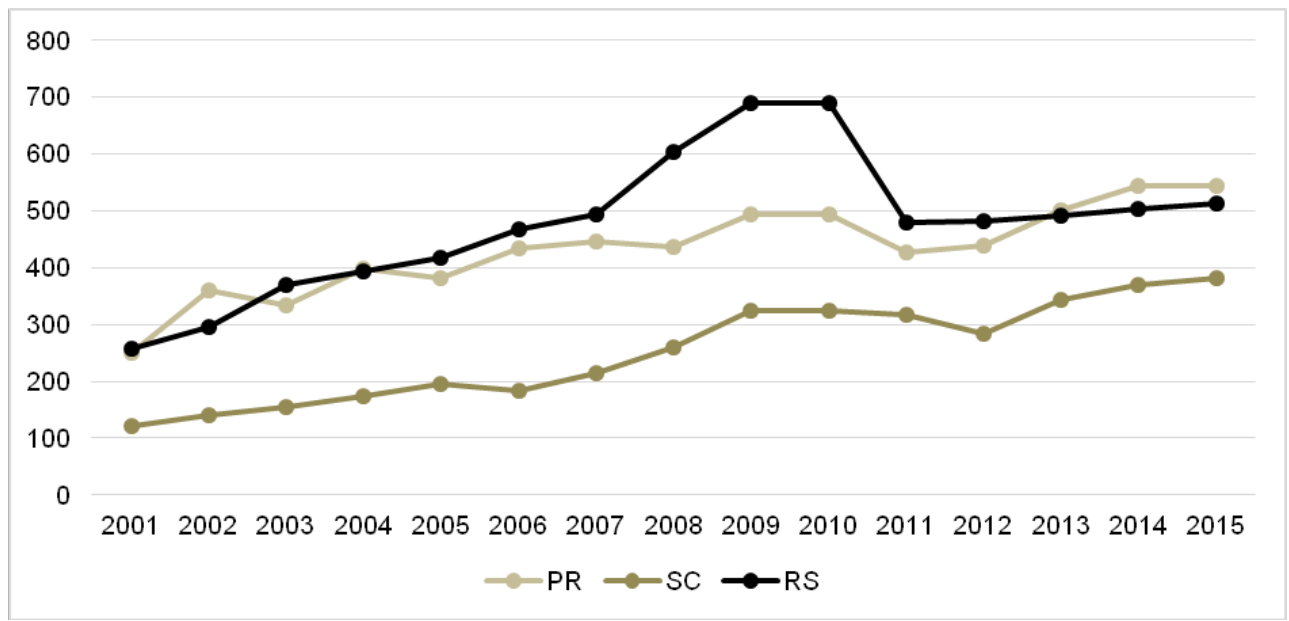

Fonte: Elaborado pelos autores com base nos dados da PNAD.

26 Na pesquisa de Vicente, Anefalos e Caser (2003) foi medido a influência do capital humano sobre a produtividade total dos fatores da agricultura brasileira durante as décadas de 1970, 1980 e meados de 1990. Os coeficientes obtidos da escolaridade foram positivos e significativos a nível nacional. No que se refere a nível regional, foram estimadas as elasticidades do índice da produção total dos fatores, e a variável escolaridade de pessoas residentes em zonas rurais foi de 0,34 , acima da média nacional que foi de 0,29 . Porém, as taxas internas de retorno da região Sul foram as menores, juntamente com a região sudeste. Mas quando se desagrega por cultura, no ano de 1996 a escolaridade dos produtores das culturas de Milho e Soja não apresentaram relevantes ganhos de produtividade da terra (Dossa et al, 1997).

27 A figura 2 apresenta a produtividade agrícola $(\mathrm{kg} / \mathrm{ha})$ das principais culturas da região Sul em forma agregada: Algodão, Batata, Café em grãos, Cana-de-açúcar, Erva-Mate, Feijão, Laranja, Maçã, Mandioca, Milho em grãos, Soja em grãos e Trigo. 0 estado de Santa Catarina por ter um menor nível de capital humano empregado em suas zonas rurais, possui a menor produtividade agrícola da região, quatro vezes menor que o estado do Rio Grande do Sul. 
Figura 2 - Produtividade agrícola das principais culturas dos estados da região Sul.

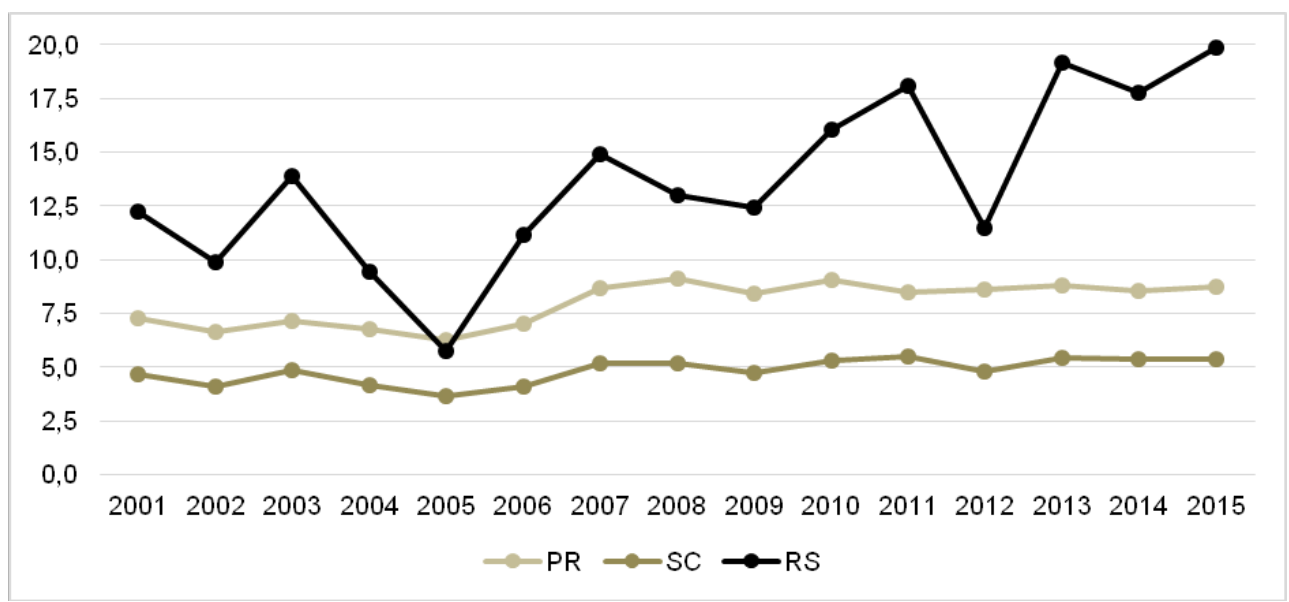

Fonte: Elaborado pelos autores com base nos dados da CONAB.

Visto a relação entre escolaridade e produção agrícola, Brigatte e Teixeira (2011) utilizaram a cointegração pelo método de Johansen para analisar os determinantes da produtividade total dos fatores da agropecuária nacional entre os anos de 1974 e 2005, e foi observado que a escolaridade tem efeito positivo não somente na produtividade total dos fatores, mas como também no produto agropecuário. Os resultados da pesquisa apontam que um aumento médio de $1 \%$ na escolaridade de trabalhadores de zonas rurais provocaria uma elevação de $1,09 \%$ na PTF e $0,60 \%$ no produto do setor agropecuário. Diante disso, se faz necessário verificar se os resultados tem o mesmo efeito para a região Sul, por causa de sua escassez de pesquisas sobre o assunto para a região.

\section{Abordagem metodologica}

29 Com o objetivo de verificar o efeito da escolaridade de pessoas que trabalham e residem nas zonas rurais dos estados da região Sul sobre a produção agrícola de seus principais produtos, foram inseridas na regressão variáveis que se adequassem ao período de estudo, onde seus dados foram retirados da Pesquisa Nacional por Amostra de Domicílios - PNAD/IBGE, Produção Agrícola Municipal - PAM/IBGE e Companhia Nacional de Abastecimento - CONAB, entre os anos de 2001 e 2015.

O método empregado na pesquisa é o de Dados em Painel, que abrange cross section e séries temporais, muitas vezes utilizado quando se possui diferentes unidades de medida ao longo do tempo. A metodologia de Dados em Painel é constantemente aplicada em pesquisas no âmbito "agro". De modo que Mendes, Teixeira e Salvato (2009) verificaram que investimentos em infraestrutura, como rodovias e irrigação, tem impacto sobre a PTF da agricultura brasileira. E também como Bressan et al (2009) utilizaram Dados em Painel para analisar os determinantes do endividamento de empresas ligadas ao agronegócio nacional, fatores como oportunidade de crescimento e lucratividade foram significativos em seus endividamentos.

Os modelos de regressão com dados em painel pode se dividir em algumas partes, como Pooled ${ }^{1}$, Efeitos Fixos e Efeitos Aleatórios. De acordo com Wooldridge (2002), a constante do modelo de Efeitos Fixos pode ter correlação com algum dos regressores, enquanto no modelo de Efeitos Aleatórios não existe essa correlação. 


$$
\operatorname{lnprodução}=\alpha+\beta \ln A P+\delta \ln A C+\theta \ln N P+\phi \ln P M F O+\psi \ln P M F Q+\varepsilon_{i}
$$

(1)

Onde lnproduçãorepresenta em logaritmo a quantidade produzida dos principais produtos agrícolas da região Sul; $\alpha$ é a constante; $\ln A P$ é o logaritmo da área plantada em hectares; $\ln A C$ é o logaritmo da área colhida em hectares; $\operatorname{lnNPé~o~logaritmo~do~}$ número de pessoas que concluíram pelo menos o ensino médio, e que residem e também trabalham em zonas rurais da região Sul; lnPMFOé o logaritmo do preço médio do fertilizante orgânico ${ }^{2}$ na região Sul; $\ln P M F Q$ é o logaritmo do preço médio do fertilizante químico na região Sul; e $\varepsilon_{i}$ é o termo de erro.

De maneira especifica o modelo de Efeitos Fixos é apresentado por Green (2002), dessa forma é possível capturar a diferença entre as unidades no termo constante:

$y_{i}=X_{i} \beta+I a_{i}+\varepsilon_{t}(2)$

Em que $X_{i}$ são as observações T para cada unidade $i$; Ié uma coluna de uns com tamanho T x 1; cada $a_{i}$ é um parâmetro desconhecido a ser estimado; e $\varepsilon_{t}$ é o termo de erro. Já o modelo de Efeitos Aleatórios pode ser estimado da seguinte forma quando os componentes da variação são desconhecidos:

$y_{i}=X_{i} \beta+\alpha+\varepsilon_{i}+u_{i}(3)$

Onde $u_{i}$ absorve essa não-observação, permitindo assim a correlação entre as variáveis incluídas e os efeitos individuais omitidos.

\section{Resultados e discussão}

34 Para a escolha do modelo realizou-se o Teste de Chow, no qual rejeitou a hipótese nula de que o modelo Pooled é o mais adequado que o de Efeitos Fixos. Em seguida, realizouse o Teste de Hausman, no qual também rejeitou-se a hipótese nula de que o modelo com Efeitos Aleatórios seria mais indicado do que o modelo com Efeitos Fixos. Pelo princípio da transitividade, como o modelo com Efeitos Fixos se mostrou mais adequado, não houve necessidade de estimar o modelo na forma empilhado, como apresenta a tabela 1.

Tabela 1 - Estimação por Dados em painel.

\begin{tabular}{|c|c|c|}
\hline Variáveis & Efeito Fixo & Efeito Aleatório \\
\hline \multirow[t]{2}{*}{$\ln \mathrm{AP}$} & -0.8310 & 0.2060 \\
\hline & $(0.47)$ & $(0.19)$ \\
\hline $\ln A C$ & $2.1229^{* *}$ & $0.6987^{* *}$ \\
\hline & $(0.43)$ & $(0.08)$ \\
\hline
\end{tabular}

O resultado de Teste de White rejeita a hipótese nula do modelo possuir heterocedasticidade, ou seja, a variação do erro é constante como explica Hoffmann (2006). Quando o modelo possui homocedasticidade, ele respeita um dos pressupostos de que:

$$
E\left[Y_{i}-E\left(Y_{i} \mid X_{i}\right)\right]^{2}=\sigma^{2}(4)
$$




$$
E\left(u_{i}^{2}\right)=\sigma^{2}(5)
$$

o fato da variável $\ln A P$ ser estatisticamente insignificante sobre a produção agrícola mas a variável $\ln A C$ ser significante à $1 \%$, pode estar relacionada à agricultura de precisão como explica Molin (2000), a agricultura de precisão faz uma junção de métodos avançados, muitas vezes demandante de mão-de-obra qualificada para monitorar a colheita, e isso torna a área colhida com mais importância do que a área plantada.

O resultado da estimação que aufere essa pesquisa é que o número de pessoas residentes e que trabalham nas zonas rurais da região Sul e possuem no mínimo o ensino médio completo, tem efeito positivo sobre a produção dos principais produtos agrícolas. Essa relação positiva se mostrou significativa tanto no modelo com Efeitos Fixos, como no modelo com Efeitos Aleatórios, embora como o modelo de Efeitos Fixos é mais adequado, a variável $\ln N P$ é significativa e positiva à $5 \%$.

Ainda no modelo de Efeitos Fixos, as variáveis $\ln P M F O$ e $\ln P M F Q$ não são estatisticamente significativas sobre a produção dos principais produtos agrícolas da região Sul. Cella e Lima Rossi (2010) explicam que o fato da agricultura brasileira ter se expandido mais rápido que a indústria de fertilizantes, fez com que a importação do insumo fosse uma solução. Portanto, o produtor pode ser muitas vezes indiferente ao preço dos fertilizantes devido à limitada oferta no país, e também a necessidade de iniciar a produção o mais rápido possível com o uso de tecnologias, pode ser uma barreira para o levantamento de preços e especulação do insumo.

\section{Conclusão}

37 A hipótese do estudo de que a escolaridade de pessoas que residem e trabalham em zonas rurais da região Sul tem efeito positivo sobre a produção de seus principais produtos agrícolas foi validada. Além da produção agrícola, a produtividade média de trabalhadores que moram em zonas rurais tende a ser maior conforme aumentar sua escolaridade, por exemplo quando o trabalhador ao menos conclui o ensino médio, mesmo que o curso não seja voltado à agricultura.

A necessidade de elevar o grau de escolaridade da mão-de-obra destinada às zonas rurais se justifica na eminência de tecnologias utilizadas na produção agrícola. Um atraso na escolaridade, inibe o nível de capital humano tornando o desenvolvimento de zonas rurais cada vez mais lento.

Assim sendo, o capital humano presente nas zonas rurais da região Sul não somente tem efeito direto sobre a produção agrícola, mas também causa efeito indireto sobre fatores como área colhida, cada vez mais repleta de tecnologia, que por sua vez acelera o ciclo de produção. Caso a mão-de-obra responsável pela produção agrícola não tenha conhecimento suficiente para controlar a tecnologia proeminente, a aceleração do processo de produção pode ser dispendiosa no sentido de tempo, fazendo com que o produtor aloque sua atenção para equalizar a falta de conhecimento necessária para utilização de máquinas e equipamentos, e portanto, tirando sua atenção de fatores também fundamentais como preços de insumos. 


\section{BIBLIOGRAFIA}

BARRO, R. J. Economic growth in a cross section of countries. The quarterly journal of economics, 1991. P. 407-443.

BRESSAN, V. G. F., et al. Análise dos determinantes do endividamento das empresas de capital aberto do agronegócio brasileiro. Revista de Economia e Sociologia Rural, 47.1, 2009. P. 89-122.

BRIGATTE, H.; TEIXEIRA, E. C. Determinantes de longo prazo do produto e da Produtividade Total dos Fatores da agropecuária brasileira no período 1974-2005. Revista de Economia e Sociologia Rural, 49.4, 2011. P. 815-836.

CABRAL, A.; SILVA, C. L. M.; SILVA, L. F. L. Teoria do capital humano, educação, desenvolvimento econômico e suas implicações na formação de professores. Revista Principia. Divulgação Científica e Tecnológica do IFPB, 32, 2016.

CELLA, D.; LIMA ROSSI, M. C. Análise do mercado de fertilizantes no Brasil. Revista Interface Tecnológica, 7.1, 2010.

CEPEA - Centro de Estudos Avançados em Economia Aplicada. PIB do Agronegócio brasileiro de 1996 a 2018. Disponível em: <https://www.cepea.esalq.usp.br/br >. Acessado em: 27/12/2018.

CEPEA - Centro de Estudos Avançados em Economia Aplicada. Mercado de Trabalho em 2017: PIB cresce, mas agronegócio tem menos ocupados. Boletim CEPEA do Mercado de trabalho, 2018. Disponível em: <https://www.cepea.esalq.usp.br/br>. Acessado em: 27/12/2018.

CONAB - Companhia Nacional de Abastecimento. Disponível em: <https://www.conab.gov.br>. Acessado em: 15/12/2018

DOSSA, D.; RODIGHERI, H. R.; CONTO, A. J. Os recursos humanos e a competitividade agrícola brasileira. In: Congresso Brasileiro de Administração Rural, 2, 1997.

FERREIRA, S.G; VELOSO, F.A. A escassez da educação. In: GIAMBIAGI, F.; E VILLELA, A. Economia Brasileira Contemporânea (1945-2004). - Rio de Janeiro: Elsevier, 2005, p.378-399.

GÓMEZ, J. T. M. Desenvolvimento em (dês) construção: Provocações e questões sobre desenvolvimento. Imaginários de desenvolvimento. Geografia Agrária-Teoria e Poder. São Paulo: Expressão Popular, 2007.

GREENE, W.H. Econometric Analysis 5th Edition. Prentice Hall. Upper Saddle River, New Jersey, 2002.

HOFFMANN, Rodolfo. Análise de Regressão: Uma introdução à econometria. 4a. ed. São Paulo: HUCITEC, 2006.

HOFFMANN, R.; GOMES NEY, M. Desigualdade, escolaridade e rendimentos na agricultura, indústria e serviços, de 1992 a 2002. Economia e Sociedade, Campinas, 13.2 (23), 2004. P. 51-79.

IBGE - Instituto Brasileiro de Geografia e Estatística. Pesquisa Nacional de Amostra a Domicílios (PNAD). Disponível em: <http://www.ibge.gov.br> . Acessado em: 13/12/2018.

IBGE - Instituto Brasileiro de Geografia e Estatística. Pesquisa Agrícola Municipal (PAM).Disponível em: <http://www.ibge.gov.br>. Acessado em: 11/12/2018.

KAGEYAMA, A. Produtividade e renda na agricultura familiar: efeitos do PRONAF-crédito. Agricultura em São Paulo, 50.2, 2003. P. 1-13. 
LUCAS, R. On the mechanics of economic development. Journal of Monetary Economics, 1988. P. 3-42.

MENDES, C. M.; PEREIRA, R. S. Educação e produtividade rural: Reflexões conceituais para uma agenda de pesquisa, 2009. Disponível em: <http://www.sober.org.br>. Acessado em: 2018.

MENDES, S. M.; TEIXEIRA, E. C.; SALVATO, M. A. Investimentos em infra-estrutura e produtividade total dos fatores na agricultura brasileira: 1985-2004. Revista Brasileira de Economia, 63.2, 2009. P. 91-102.

MOLIN, J.P. Geração e interpretação de mapas de produtividade para agricultura de precisão. In: BORÉM, A. et al.(Ed.). Agricultura de precisão. Viçosa: Editora UFV, 2000.

MONTENEGRO, J. R. Desenvolvimento em (des) construção: provocações e questões sobre desenvolvimento e geografia. Geografía agraria: teoria e poder, Editora Expressão Popular, São Paulo, Brasil, 2007. P. 39-54.

SCHULTZ, T. W. The value of children: an economic perspective. Journal of Political Economy, $81.2,1973$.

SILVA, M. A. M. Reestruturação Produtiva e os impactos sobre os migrantes. Una Nueva Ruralidade em América Latina, 2001. P. 135-55.

STADUTO, J. A.; SHIKIDA, P. F.; BACHA, C. J. Alteração na composição da mão-de-obra assalariada na agropecuária brasileira. Agricultura em São Paulo, 51.2, 2004. P. 57-70.

STULP, V.J. Efeitos dos setores econômicos e da escolaridade sobre o rendimento do trabalho no Rio Grande do Sul. Rev. Econ. Sociol. Rural, 44.1, 2006.

VICENTE, J. R.; ANEFALOS, L.C.; CASER, D.V. Influência de capital humano, insumos modernos e recursos naturais na produtividade agrícola. Região e espaço no desenvolvimento agrícola brasileiro. Rio de janeiro: Ipea, 2003. P. 265-295.

WOOLDRIDGE, J. Econometric analysis of cross section and panel data. MIT Press, 2002.

\section{NOTAS}

1. Não houve necessidade de estimar o modelo Pooled porque o resultado do Teste de Chow foi significativo a $1 \%$.

2. Cama Aviaria, Esterco de Curral, Esterco Suíno e demais materiais orgânicos.

\section{RESUMOS}

Com o avanço de tecnologias destinadas à produção agrícola, se faz necessário cada vez mais a alocação de mão-de-obra qualificada no campo. Sendo assim, o presente estudo tem como objetivo analisar o efeito do capital humano situado em zonas rurais dos estados da região Sul sobre a produção de seus principais produtos agrícolas. Foram utilizados dados da Pesquisa Nacional por Amostra de Domicílios (PNAD), Produção Agrícola Municipal (PAM) e Companhia Nacional de Abastecimento (CONAB) para período entre 2001 e 2015. O método empregado na 
pesquisa foi o de Dados em Painel, no qual o modelo de Efeitos Fixos se mostrou mais adequado. 0 número de pessoas que possuem no mínimo o ensino médio completo, e que residem e também trabalham em zonais rurais dos estados da região Sul apresentou-se significativamente e positivamente relacionada com a produção dos principais produtos agrícolas da região.

With the advancement of technologies for agricultural production, it is increasingly necessary to allocate skilled labor in the field. Thus, the present study aims to analyze the effect of human capital located in rural areas of the southern states on the production of their main agricultural products. Data from the National Household Sample Survey (PNAD), Municipal Agricultural Production (PAM) and National Supply Company (CONAB) were used for the period between 2001 and 2015. The method used in the research was the Data in Panel, in which the model was more appropriate. The number of people with at least high school education who reside and also work in rural areas of the southern states has been significantly and positively related to the production of the region's main agricultural products.

Con el avance de las tecnologías para la producción agrícola, la asignación de mano de obra calificada en el campo es cada vez más necesaria. Por lo tanto, el presente estudio tiene como objetivo analizar el efecto del capital humano ubicado en las zonas rurales de los estados del sur en la producción de sus principales productos agrícolas. Se utilizaron datos de la Encuesta Nacional de Muestra de Hogares (PNAD), Producción Agrícola Municipal (PAM) y Empresa Nacional de Abastecimiento (CONAB) para el período comprendido entre 2001 y 2015. El método utilizado en la encuesta fue el Panel de Datos, en el cual El modelo de efectos fijos era más apropiado. El número de personas que al menos completaron la escuela secundaria, que viven y trabajan en las zonas rurales de los estados del sur se relacionó de manera significativa y positiva con la producción de los principales productos agrícolas de la región.

Avec l'avancement des technologies de production agricole, l'affectation de main-d'œuvre qualifiée dans le domaine devient de plus en plus nécessaire. Ainsi, la présente étude vise à analyser l'effet du capital humain situé dans les zones rurales des États du sud sur la production de leurs principaux produits agricoles. Les données de l'Enquête nationale par sondage auprès des ménages (PNAD), de la Production agricole municipale (PAM) et de la National Supply Company (CONAB) pour la période entre 2001 et 2015 ont été utilisées. La méthode utilisée dans l'enquête était les données de panel, dans lesquelles le Le modèle d'effets fixes était plus approprié. Le nombre de personnes qui ont au moins terminé leurs études secondaires, qui vivent et travaillent également dans les zones rurales des États du sud était significativement et positivement lié à la production des principaux produits agricoles de la région.

\section{ÍNDICE}

Mots-clés: zones rurales; production agricole; capital humain.

Keywords: rural areas; agricultural production; human capital

Palabras claves: zonas rurales; producción agrícola; capital humano

\section{AUTOR}

\section{AMARILDO DE PAULA JUNIOR}

Graduado em Economia pela UNIFCV (2017).

Mestrando em Teoria Econômica pela UEM (2018), com bolsa CAPES 